DOI: $10.21105 /$ joss.03653

\section{Software}

- Review ¿

- Repository ca

- Archive ct

Editor: Pierre de Buyl ๘ Reviewers:

- @lorenzo-rovigatti

- @junghans

Submitted: 27 June 2021 Published: 28 September 2021

\section{License}

Authors of papers retain copyright and release the work under a Creative Commons Attribution 4.0 International License (CC BY 4.0).

\title{
SiSyPHE: A Python package for the Simulation of Systems of interacting mean-field Particles with High Efficiency
}

\section{Antoine $\mathrm{Diez}^{1}$}

1 Department of Mathematics, Imperial College London, South Kensington Campus, London, SW7 2AZ, UK

\section{Summary}

Over the past decades, the study of systems of particles has become an important part of many research areas, from theoretical physics to applied biology and computational mathematics. One of the main motivations in mathematical biology is the modelling of large animal societies and the emergence of complex patterns from simple behavioral rules, e.g., flocks of birds, fish schools, ant colonies, etc. In the microscopic world, particle systems are used to model a wide range of phenomena, from the collective motion of spermatozoa to the anarchical development of cancer cells. Within this perspective, there are at least three important reasons to conduct large scale computer simulations of particle systems. First, numerical experiments are essential to calibrate the models and test the influence of each parameter in a controlled environment. For instance, the renowned Vicsek model (Vicsek et al., 1995) is a minimal model of flocking, which exhibits a complex behavior, studied numerically in particular in (Chaté et al., 2008). Secondly, particle simulations are used to check the validity of macroscopic models that describe the statistical behavior of particle systems. These models are usually based on partial differential equations (PDE) derived using phenomenological considerations that are often difficult to justify mathematically (Degond et al., 2021; Degond \& Motsch, 2008; Dimarco \& Motsch, 2016). Finally, inspired by models in biology, there is an ever growing literature on the design of algorithms based on the simulation of artificial particle systems to solve tough optimization problems (Grassi \& Pareschi, 2020; Kennedy \& Eberhart, 1995; Pinnau et al., 2017; Totzeck, 2021) and to construct new more efficient Markov Chain Monte Carlo methods (Cappé et al., 2004; Clarté et al., 2021; Del Moral, 1998, 2013; Doucet et al., 2001). The simulation of systems of particles is also at the core of molecular dynamics (Leimkuhler \& Matthews, 2015), although the present library is not specifically written for this purpose. The SiSyPHE library builds on recent advances in hardware and software for the efficient simulation of large scale interacting mean-field particle systems, both on the GPU and on the CPU. The versatile object-oriented Python interface of the library is designed for the simulation and comparison of new and classical many-particle models of collective dynamics in mathematics and active matter physics, enabling ambitious numerical experiments and leading to novel conjectures and results.

\section{Statement of need}

A major difficulty in the simulation of systems of particles is the high computational cost, typically quadratic in the number of particles, which prevents large scale experiments. The implementation of SiSyPHE is based on recent libraries originally developed for machine learning purposes to significantly accelerate tensor (array) computations, namely the PyTorch 
package (Paszke et al., 2019) and the KeOps library (Charlier et al., 2021). On a GPU, the SiSyPHE library speeds up both traditional Python and low-level implementations by one to three orders of magnitude for systems with up to several millions of particles.

In addition, to the best of our knowledge, only model-specific packages such as Motsch (2016) are available. The SiSyPHE library includes, within a common framework, the implementation of many classical models and their variants as well as recent models for which no implementation was previously available. All the models detailed in the Example gallery of the documentation are directly taken from the literature on collective dynamics in mathematics and active matter physics. Moreover, the SiSyPHE library is designed in such a way that new custom models can easily be added in order to facilite the study and comparison of models from a research perspective.

The development of the SiSyPHE library was initially motivated by the study of body-oriented particles (Degond et al., 2019). The (formal) derivation of a macroscopic PDE model from the particle system has lead to a novel conjecture which postulates the existence of a class of so-called bulk topological states in (Degond et al., 2021). The quantitative comparison between this theoretical prediction and the numerical simulation of the particle system in a suitable regime (with more than $10^{6}$ particles) has confirmed the existence of these new states of matter. The study of their physical properties which are observed in the numerical experiments but not readily explained by the PDE model is an ongoing work.

\section{A typical example}

A typical model that is implemented in the SiSyPHE library is the variant of the Vicsek model introduced by Degond \& Motsch (2008) and defined by the system of $2 N$ Stratonovich Stochastic Differential Equations

$$
\mathrm{d} X_{t}^{i}=c_{0} V_{t}^{i} \mathrm{~d} t, \quad \mathrm{~d} V_{t}^{i}=\sigma \mathrm{P}\left(V_{t}^{i}\right) \circ\left(J_{t}^{i} \mathrm{~d} t+\mathrm{d} B_{t}^{i}\right),
$$

where the position at time $t$ of a particle indexed by $i \in\{1, \ldots, N\}$ is a vector $X_{t}^{i} \in \mathbb{R}^{d}$ and its orientation (or velocity) is a unit vector $V_{t}^{i} \in \mathbb{R}^{d}$ with $\left|V_{t}^{i}\right|=1$. The coefficient $c_{0}>0$ is the speed of the particles (assumed to be constant), the matrix $\mathrm{P}\left(V_{t}^{i}\right)=I_{d}-V_{t}^{i} \otimes V_{t}^{i}$ is the orthogonal projection matrix on the plane orthgonal to $V_{t}^{i},\left(B_{t}^{i}\right)_{t}$ is an independent Brownian motion, and $\sigma>0$ is a diffusion coefficient which models the level of noise. The quantity $J_{t}^{i} \in \mathbb{R}^{d}$ is called a target; it is the orientation that particle $i$ is trying to adopt. In the Vicsek model introduced by Degond \& Motsch (2008),

$$
J_{t}^{i}=\frac{\sum_{j=1}^{N} K\left(\left|X_{t}^{j}-X_{t}^{i}\right|\right) V_{t}^{j}}{\left|\sum_{j=1}^{N} K\left(\left|X_{t}^{j}-X_{t}^{i}\right|\right) V_{t}^{j}\right|},
$$

where the kernel $K:[0,+\infty) \rightarrow[0,+\infty)$ is a smooth nonnegative function vanishing at infinity which models the visual perception of the particles; in the Vicsek model, the vision of the particles depends on the distance between them. With the target given by Equation 2, each particle tries to adopt the average orientation of its neighbors, which is a typical flocking behavior.

On a computer, the time-continuous system given by Equation 1 needs to be discretized first. For the Vicsek model, a natural discretization method is the (geometric) Euler-Maruyama scheme (Kloeden \& Platen, 1992; Piggott \& Solo, 2016). In general, the discretization method depends on the model considered as illustrated in the Example gallery. Then, at each time step, the most expensive operation is the computation of the target given by Equation 2, which requires $\mathcal{O}(N)$ operations for each of the $N$ particles. The total simulation cost is thus $\mathcal{O}\left(N^{2} T\right)$ where $T$ is the total number of iterations. Within the framework of the KeOps library on which SiSyPHE is based, the computation of the target Equation 2 is called a 
kernel operation, which is efficiently carried out using a symbolic definition of the $N \times N$ interaction matrix whose $(i, j)$-entry is $K\left(\left|X_{t}^{j}-X_{t}^{i}\right|\right)$. The computation of the target is then understood as a symbolic matrix-vector product between the interaction matrix and the vector of orientations.

\section{Acknowledgements}

The development of this library would not have been possible without the help of Jean Feydy, his constant support and precious advice. This project was initiated by Pierre Degond and has grown out of many discussions with him.

\section{References}

Cappé, O., Guillin, A., Marin, J. M., \& Robert, C. P. (2004). Population Monte Carlo. J. Comput. Graph. Statist., 13(4), 907-929. https://doi.org/10.1198/106186004X12803

Charlier, B., Feydy, J., Glaunès, J. A., Collin, F.-D., \& Durif, G. (2021). Kernel Operations on the GPU, with Autodiff, without Memory Overflows. J. Mach. Learn. Res., 22(74), 1-6.

Chaté, H., Ginelli, F., Grégoire, G., \& Raynaud, F. (2008). Collective motion of self-propelled particles interacting without cohesion. Phys. Rev. E, 77(4), 046113. https://doi.org/10. 1103/PhysRevE.77.046113

Clarté, G., Diez, A., \& Feydy, J. (2021). Collective Proposal Distributions for Nonlinear MCMC samplers: Mean-Field Theory and Fast Implementation. arXiv Preprint: arXiv:1909.08988. http://arxiv.org/abs/1909.08988

Degond, P., Diez, A., \& Na, M. (2021). Bulk topological states in a new collective dynamics model. arXiv Preprint: arXiv:2101.10864. http://arxiv.org/abs/2101.10864

Degond, P., Frouvelle, A., Merino-Aceituno, S., \& Trescases, A. (2019). Alignment of Selfpropelled Rigid Bodies: From Particle Systems to Macroscopic Equations. In G. Giacomin, S. Olla, E. Saada, H. Spohn, \& G. Stoltz (Eds.), Stochastic Dynamics Out of Equilibrium, Institut Henri Poincaré, Paris, France, 2017 (pp. 28-66). Springer, Cham. https://doi. org/10.1007/978-3-030-15096-9_2

Degond, P., \& Motsch, S. (2008). Continuum limit of self-driven particles with orientation interaction. Math. Models Methods Appl. Sci., 18(Suppl.), 1193-1215. https://doi.org/ $10.1142 /$ S0218202508003005

Del Moral, P. (1998). Measure-valued processes and interacting particle systems. Application to nonlinear filtering problems. Ann. Appl. Probab., 8, 438-495. https://doi.org/10. $1214 /$ aoap/1028903535

Del Moral, P. (2013). Mean field simulation for Monte Carlo integration. CRC Press, Taylor \& Francis Group. ISBN: 9781466504059

Dimarco, G., \& Motsch, S. (2016). Self-alignment driven by jump processes: Macroscopic limit and numerical investigation. Math. Models Methods Appl. Sci., 26(07), 1385-1410. https://doi.org/10.1142/S0218202516500330

Doucet, A., Freitas, N., \& Gordon, N. (Eds.). (2001). Sequential Monte Carlo Methods in Practice. Springer-Verlag New York. https://doi.org/10.1007/978-1-4757-3437-9

Grassi, S., \& Pareschi, L. (2020). From particle swarm optimization to consensus based optimization: Stochastic modeling and mean-field limit. Math. Models Methods Appl. Sci., 31(8), 1625-1657. https://doi.org/10.1142/s0218202521500342 
Kennedy, J., \& Eberhart, R. (1995). Particle swarm optimization. Proceedings of ICNN'95 - International Conference on Neural Networks, 4, 1942-1948. https://doi.org/10.1109/ ICNN.1995.488968

Kloeden, P. E., \& Platen, E. (1992). Numerical solution of stochastic differential equations. Springer, Berlin, Heidelberg. https://doi.org/10.1007/978-3-662-12616-5

Leimkuhler, B., \& Matthews, C. (2015). Molecular dynamics with deterministic and stochastic numerical methods. Springer International Publishing. https: //doi.org/10.1007/978-3-319-16375-8

Motsch, S. (2016). Vicsek_microFlat. GitHub Repository. https://github.com/smotsch/ Vicsek_microFlat

Paszke, A., Gross, S., Massa, F., Lerer, A., Bradbury, J., Chanan, G., Killeen, T., Lin, Z., Gimelshein, N., Antiga, L., Desmaison, A., Kopf, A., Yang, E., DeVito, Z., Raison, M., Tejani, A., Chilamkurthy, S., Steiner, B., Fang, L., ... Chintala, S. (2019). PyTorch: An Imperative Style, High-Performance Deep Learning Library. In H. Wallach, H. Larochelle, A. Beygelzimer, F. Alché-Buc, E. Fox, \& R. Garnett (Eds.), Advances in Neural Information Processing Systems (Vol. 32). Curran Associates, Inc. https://proceedings.neurips.cc/ paper/2019/file/bdbca288fee7f92f2bfa9f7012727740-Paper.pdf

Piggott, M. J., \& Solo, V. (2016). Geometric Euler-Maruyama schemes for stochastic differential equations in SO(n) and SE(n). SIAM J. Numer. Anal., 54(4), 2490-2516. https://doi.org/10.1137/15M1019726

Pinnau, R., Totzeck, C., Tse, O., \& Martin, S. (2017). A consensus-based model for global optimization and its mean-field limit. Math. Models Methods Appl. Sci., 27(01), 183204. https://doi.org/10.1142/S0218202517400061

Totzeck, C. (2021). Trends in Consensus-based optimization. arXiv Preprint: arXiv:2104.01383. http://arxiv.org/abs/2104.01383

Vicsek, T., Czirók, A., Ben-Jacob, E., Cohen, I., \& Shochet, O. (1995). Novel Type of Phase Transition in a System of Self-Driven Particles. Phys. Rev. Lett., 75(6), 1226-1229. https://doi.org/10.1103/PhysRevLett.75.1226 